\title{
Identité disciplinaire : éclatement ou cohérence
}

\section{Annie Rouxel}

\section{OpenEdition}

Journals

Édition électronique

URL : http://journals.openedition.org/trema/1580

DOI : 10.4000/trema. 1580

ISSN : 2107-0997

\section{Éditeur}

Faculté d'Éducation de l'université de Montpellier

\section{Édition imprimée}

Date de publication : 1 octobre 2002

Pagination : 29-36

ISSN : 1167-315X

\section{Référence électronique}

Annie Rouxel, «Identité disciplinaire : éclatement ou cohérence », Tréma [En ligne], 19 | 2002, mis en ligne le 01 octobre 2002, consulté le 01 mai 2019. URL : http://journals.openedition.org/trema/1580 ; DOI : 10.4000/trema.1580

Ce document a été généré automatiquement le 1 mai 2019.

Trema 


\title{
Identité disciplinaire : éclatement ou cohérence
}

\author{
Annie Rouxel
}

\begin{abstract}
« L'enjeu essentiel de l'enseignement du français aujourd'hui semble résider dans cette perspective : comment, face à l'éclatement, la dispersion des savoirs, faire de l'enseignement du français une réorganisation d'un savoir éclaté ? L'introduction de supports actuels dans le cadre de l'enseignement du français, à côté de textes plus classiques comme l'illustre l'organisation de ce manuel sous-tend l'évolution du français (entre transmission de savoirs et compréhension du
\end{abstract} monde). »

\section{Un constat empirique}

1 Ainsi s'exprimait en décembre dernier une étudiante de licence de l'université de Rennes II, dans le cadre de l'UED « Sensibilisation aux métiers de l'enseignement, second degré ». J'avais demandé aux étudiants de réfléchir à la discipline "français » (traits définitoires et évolution) à partir de la table des matières d'un manuel de troisième ${ }^{1}$.

2 Ce qu'ont clairement perçu ces étudiants, c'est non seulement l'écart entre la discipline telle qu'elle apparaît aujourd'hui dans ce manuel, avec ses nouveaux objets, notamment l'image et l'oral, et ce qu'elle était dans le passé (et dans leur passé d'élève) mais aussi l'écart qu'elle présente avec ce qu'elle est dans leur expérience quotidienne à l'université. Diversité, ouverture, hétérogénéité, extension des frontières, éclatement, dilution dans la transversalité... Tels sont les termes utilisés pour saisir et interroger la forme actuelle de la discipline au collège. Crise de l'identité disciplinaire ou complexification dont la cohérence échappe au premier abord? Réflexion essentielle chez des étudiants qui 
envisagent de devenir enseignants et dont l'une des premières motivations est de transmettre leur passion pour la littérature.

\section{Un pluriel déjà interrogé}

3 Mais, pour la communauté didactique, cette question n'est pas nouvelle. La pluralité des objets disciplinaires, des savoirs de référence et des pratiques a maintes fois été évoquée dans la dernière décennie. En 1995, l'ouvrage de Jean-Louis Chiss, Jacques David et Yves Reuter Didactique du français, État d'une discipline interrogeait déjà la pertinence du mot didactique au singulier pour rendre compte de la complexité du champ disciplinaire. Les deux colloques de la DFLM, à partir de leurs problématiques spécifiques - Les métalangages de la classe de français, en 1995 et Quels savoirs pour quelles valeurs ? en 1998 - ont également abordé la question de l'hétérogénéité disciplinaire. Enfin, le colloque de Toulouse Enjeux didactiques des théories du texte dans l'enseignement du français, en 1998, en faisant le bilan des théories du texte et en dressant l'inventaire des savoirs utiles à la lecture des textes littéraires pointait également le pluriel qui nous intéresse aujourd'hui.

\section{3. Évolutions, réformes structurelles et matrice disciplinaire}

4 La discipline change dans ses contenus ses objectifs et ses finalités. L'évolution diachronique est, on le sait, commandée par les transformations de la société (demande sociale et volonté politique) et par le développement des connaissances scientifiques. Nouveaux publics, nouveaux savoirs... Mais ces changements n'affectent pas de la même façon tous les niveaux et l'observation de la discipline, dans une perspective synchronique et longitudinale, de la maternelle à l'université, oblige à s'interroger sur la pertinence même de la notion de discipline pour décrire ce que l'on désigne ordinairement par « enseignement du français ».

5 Appréhendée comme une évidence, cette notion unificatrice et commode recouvre en réalité des objets et des pratiques très hétérogènes. Selon Michel Develay ${ }^{2}$, une discipline se définit comme un ensemble composite de connaissances déclaratives et procédurales qui s'actualisent dans des tâches et visent des objectifs. C'est la matrice disciplinaire, principe organisateur où se lisent les choix axiologiques, qui assure la cohérence de l'ensemble.

6 Or, si on considère l'ensemble de l'enseignement du français, ce qui frappe au premier abord, c'est une telle disparité des formes prises par la configuration disciplinaire aux différents niveaux que l'on peut s'interroger sur l'existence d'une matrice disciplinaire unique. Si le pluriel est consubstantiel à l'identité du français ne serait-ce que par la diversité de ses objets - langue, texte, discours, littérature, image - des savoirs de référence qu'il convoque, des objectifs qu'il vise et des activités multiples auxquelles il donne lieu, la question qui se pose est bien celle-ci : comment accueillir cette diversité sans perdre de vue ce qui la fonde légitimement? Comment la penser pour lui donner du sens ? Comment percevoir à travers chaque activité dans sa singularité ce qui la rattache au socle commun de la discipline? 
7 Du collège au lycée, ce qui transcende le pluriel des objectifs, des contenus et des activités, c'est la maîtrise des discours : discours de la communication ordinaire, discours des médias, discours littéraire, discours de l'image.

8 Le collège tente de mettre en cohérence les apprentissages au sein des séquences en décloisonnant les composantes constitutives de la discipline, en évitant de leur assigner des horaires précis, en mettant l'accent sur la complémentarité des savoirs. Toutefois, dans la hiérarchie des domaines disciplinaires étudiés, c'est le volet étude de la langue qui prime sur l'approche littéraire des textes souvent limitée à la compréhension; et pour ce qui concerne les supports d'étude, le collège apparaît comme le lieu de l'ouverture maximale.

9 Au lycée, le poids respectif des composantes disciplinaires s'inverse ; l'étude de la langue s'estompe au profit de la littérature. C'est avant tout le discours littéraire avec ses spécificités qui est étudié.

10 En dépit des variations internes au champ disciplinaire, la notion de matrice disciplinaire est pertinente pour décrire le français enseigné dans le second degré.

Qu'en est-il en amont et en aval?

11 En premier lieu, on peut se demander si la notion même de discipline est pertinente pour le premier degré où les apprentissages fondamentaux - lire et écrire - relèvent très souvent d'enseignants non spécialistes ${ }^{3}$ et s'effectuent à chaque instant au cœur d'autres matières. La vocation utilitaire et transversale du français est affirmée. La place de la littérature est seconde et concerne presque essentiellement la littérature de jeunesse.

12 À l'université enfin, la discipline réduit fortement son champ et ses composantes: littérature française, littérature comparée et linguistique ont le contour net de spécialités qui ne communiquent pas. Quant à l'image, elle reste aux marges de la discipline, convoquée par les rares enseignants qui s'en sont fait une spécialité. Il est par exemple assez paradoxal de voir que l'ouvrage de Patrick Née ${ }^{4}$ consacré à la lecture littéraire de Nadja de Breton, ne s'intéresse pas à l'insertion des quarante-huit photographies dans le texte.

Image fluctuante de la discipline donc, au sein de laquelle la place et le statut de la langue, de la littérature et de l'image présentent des variations importantes et problématiques. À l'évidence la matrice disciplinaire définie par la maitrise des discours n'est pas pertinente pour décrire les deux extrémités des cursus, école primaire et université. Elle ne concerne que l'enseignement secondaire dont elle souligne la continuité entre collège et lycée.

Cette dissemblance de la discipline dans les quatre niveaux se traduit schématiquement par l'inversion du poids respectif de la langue et de la littérature. La nécessité de mettre en avant la continuité s'exprime par les réformes structurelles (cycle 3 et $\mathrm{CP}$ ) et les dispositifs de liaison inter-cycles (CM2-6, troisième-seconde) qui entérinent le glissement de la dominante langue à la dominante la littérature.

La mise en place des réformes à l'école primaire et à l'université, modifie la donne et rend plus aigu aujourd'hui ce problème de l'identité disciplinaire.

16 C'est au cycle 3 de l'école primaire que la transformation radicale des structures de la discipline est la plus flagrante : non plus juxtaposition de composantes entre lesquelles on tente d'établir des liens au fil des apprentissages pour les motiver les unes par les autres (lecture/écriture; grammaire/orthographe; grammaire/lecture ; écriture/orthographe et grammaire) mais véritable redistribution et même scission, partition entre la maîtrise 
du langage d'une part et la littérature et la grammaire d'autre part, désormais toutes deux composantes de "l'éducation littéraire et humaine ". Cependant, cette division, en rupture brutale avec nos représentations de la discipline, n'introduit-elle pas une certaine cohérence au plan épistémologique en refondant dès le primaire la discipline dans les domaines où elle se déploie dans la suite du cursus?

Quant à la réforme prévue pour l'université de demain, elle détermine des parcours-types susceptibles de nombreuses combinatoires qui mettent fin à la spécialisation disciplinaire telle qu'elle existe aujourd'hui.

Il est sans doute prématuré de porter un jugement sur des restructurations qui n'ont pas encore vu le jour et qui s'annoncent en rupture avec l'existant puisqu'elles font voler en éclats les contours traditionnels de la discipline aux deux bouts du cursus. Et pourtant, les questions : rupture ou continuité ? Éclatement ou cohérence ? Continuent de se poser car il est certain que les éléments de cohérence et de continuité ne résident pas seulement dans les structures.

\section{Mettre au jour les fils invisibles de la formation culturelle et humaine}

Il n'est pas certain en effet que la reconfiguration de l'enseignement du français au cycle 3 de l'école primaire et que le projet pour le supérieur n'introduisent pas un peu de cohérence propre à revitaliser la discipline.

20 Au-delà des découpages institutionnels, les vraies interrogations concernant notre discipline portent en effet sur les enjeux en termes de formation. C'est à ce niveau que peut se jouer la cohérence, lors même que les champs disciplinaires ne coïncident pas.

21 Pour apporter quelques éléments de réflexion sur cette question, je limiterai mon propos à un exemple, celui de l'élaboration des compétences de lecture, ce qui oblige dans la foulée à considérer le statut du texte et de l'image.

\section{Un constat partagé : le couple comprendre/interpréter partagé entre collège et lycée}

22 À l'école primaire aujourd'hui, l'approche de la lecture est orientée vers la compréhension. "Lire, c'est comprendre » déclare-t-on dans les textes officiels et les méthodes de lecture du Cours préparatoire en maintenant le flou épistémologique sur ce que signifie comprendre. Du reste, peut-on lire sans comprendre ? Ce qui se joue à ce niveau c'est le rapport de l'élève au texte, au monde et à la culture. Or, qu'est-ce que lire quand on apprend à lire sur des artefacts conçus pour les acquisitions phonologiques? Qu'est-ce que lire quand l'image se substitue au texte et fait obstacle à l'activité figurative de l'élève? Quel monde présente-t-on dans les méthodes sinon, comme l'a montré Luc Maisonneuve dans la thèse qu'il vient de soutenir ${ }^{5}$, l'univers stéréotypé des séries et de la publicité.

23 Au-delà du cours préparatoire, c'est une littérature adaptée à l'âge des enfants - albums, $\mathrm{BD}$, contes, romans - qui est proposée pour encourager la lecture, notamment en lien avec les BCD. Mais en classe, lire revient bien souvent à élucider le vocabulaire pour accéder au sens littéral et répondre à des questions qui le vérifient. Marie-Thérèse Denizeau $^{6}$ et Catherine Tauveron ${ }^{7}$ ont déjà décrit le rituel stérile de ces pratiques de classe, je n'y reviens pas. 
24 Au collège, c'est encore la lecture - compréhension qui domine l'approche du corpus de littérature large et diversifié proposé aux élèves. Entre repérages et reformulation, il y a peu de place pour une construction personnelle de l'élève comme lecteur d'autant que le formalisme habituellement évoqué pour le lycée y règne souvent en maître. Approches narratologiques qui confondent l'outil et l'objet, lecture de l'image ${ }^{8}$ aléatoire dépendante de l'initiative de l'enseignant - et subordonnée au texte, image illustrative le plus souvent, qui n'apporte souvent ni enrichissement du sens ni gain en termes de formation.

25 Au lycée, de longue tradition, on considère que l'élève est en mesure d'interpréter un texte. En témoignent l'exercice du commentaire littéraire et la certification du baccalauréat.

Les nouveaux programmes de lycée qui se mettent en place aujourd'hui renforcent cette attente. Ils instaurent l'élève comme lecteur et construisent ses compétences interprétatives. L'approche des textes littéraires et des images par les registres conçus comme des postures anthropologiques, des catégories de perception du monde, donne sens à la lecture. Elle permet aux élèves de tirer parti de l'expérience humaine, de percevoir la littérature non plus comme un empilement de textes à connaître mais comme un moyen de mieux comprendre le monde. La lecture cursive introduit un nouveau rapport au texte et développe les compétences de lecture en contribuant à l'élaboration d'une culture personnelle.

Mais il ne suffit pas de le décréter. Encore faut-il que cette volonté institutionnelle passe dans les faits.

\section{Les nouveaux programmes source de cohérence ? Comprendre/interpréter : un continuum tout au long du cursus}

27 L'introduction de la littérature à l'école primaire n'a de sens que si l'on admet la capacité des jeunes élèves à interpréter un texte et si on encourage les démarches interprétatives. Les textes littéraires, surtout lorsqu'ils résistent à la compréhension immédiate, se prêtent particulièrement à la formation des lecteurs, la complexité stimulant des démarches heuristiques. Les recherches de Catherine Tauveron et Patrick Demougin, entre autres, ont montré que les potentialités des jeunes élèves restent trop souvent en friches actuellement. Ne pas attendre le lycée pour effectuer le saut qualitatif est un enjeu fort qui repose sur un choix épistémologique et éthique qu'il conviendrait d'aborder en formation. Alors peut-être pourrait-on parler de cohérence dans la formation en dépit des différences de corpus.

28 La logique d'un tel changement devrait se poursuivre au collège avec les perspectives offertes par l'étude en sixième des textes fondateurs. Le choix du corpus littéraire est déterminant dans la formation des élèves. Découvrir les grands univers mythiques, c'est nécessairement apprendre à interpréter les usages métaphoriques du langage. Ces textes qui parlent de l'expérience humaine permettent aux élèves de construire leur propre identité tout en accédant aux références culturelles nécessaires à la lecture de nombreux textes et images. Sur ce point, je renvoie aux recherches de Marie-josé Fourtanier'. C'est la question du sens qui est posée : sens des textes pour que l'école ait du sens. 
nécessitent des prises de conscience et des formations.

Au bout du parcours, l'université a tout à gagner de ce travail en amont.

J'ai suivi le fil «compétence de lecture » pour repérer disjonction ou continuité, en faisant l'hypothèse que la cohérence est plutôt du côté de la continuité. (Ce qui ne signifie pas qu'il existe une relation obligée entre les deux termes, la rupture pouvant être cohérente à un moment donné d'une progression. C'est en réalité le choix épistémologique qui a guidé l'analyse). Il faudrait maintenant tirer le fil des compétences d'écriture puis celui la maîtrise de la langue. Deux colloques y consacrent leurs travaux, celui de l'INRP la semaine dernière, celui de l'AFEF en mai prochain.

La conclusion provisoire à laquelle je parviens, c'est que «extension du champ disciplinaire " ne signifie pas " éclatement ", que l'éclatement se repère à la perte de sens qu'il révèle ou occasionne. Que les composantes disciplinaires soient plus nombreuses devient au contraire un enrichissement si du point de vue de la formation elles opèrent dans le même sens. (Car il faut à la fois considérer la cohérence entre les composantes disciplinaires et la cohérence interne à chacune). La porosité des frontières disciplinaires est un faux problème : les découpages disciplinaires ont leur raison d'être moins en euxmêmes que dans la tradition culturelle d'un pays. Le rêve de d'Alembert appartient autant à la littérature qu'à la philosophie, le "J'accuse " de Zola autant à la littérature qu'à l'histoire. La richesse des textes résiste à la tendance française à la classification.

Mais dans la perspective de recomposition et d'élargissement du champ disciplinaire qui caractérise notre époque, où s'arrêter ? Pourquoi, par exemple, y inclure l'image et laisser à l'écart ce qui sous-tend et modèle les littératures orales, à savoir les rythmes et musiques qui les font naître et les accompagnent. Il y a deux ans, notre collègue malien Sékou Biabaté, soulignait l'importance de la musique liée à la diction de l'épopée dans son pays. Mais la question se pose aussi dans notre tradition littéraire française puisqu'au moyen âge et au seizième siècle, la poésie était chantée... Au moment où l'on tente d'ébranler la tradition scolaire graphocentrique, pourquoi ne pas introduire aussi musique et rythme qui sont la mémoire des textes et donnent forme aux discours?

Quelle place donner au théâtre vivant qui reste, dans la pratique scolaire, trop souvent aussi aux marges de la discipline?

\section{Trois points pour conclure}

1. Pas d'éclatement ; une cohérence masquée qu'il faut rendre explicite en formation La tendance à l'ouverture, que l'on constate aujourd'hui, est intéressante entre autres parce qu'elle suscite la réflexion et encourage la recherche de convergences intra-disciplinaires (entre les composantes de la discipline).

2. Cette tendance à l'ouverture commence à se manifester à l'université, lieu où prévaut d'ordinaire la défense des spécialités. Elle s'amorce sous la forme d'approches croisées interdisciplinaires, d'interrogations sur les rapports entre différents systèmes sémiotiques. Par exemple, à Rennes, la tenue récente d'un séminaire interdisciplinaire sur Proust et la photographie à partir des commentaires de Brassai sur La Recherche du temps perdu.

3. Une des spécificités du français, c'est d'être une discipline aux contours mouvants, instables, une discipline marquée par la variabilité historique. Les évolutions rapides obligent à travailler dans l'inconfort du provisoire, de l'aléatoire. Mais c'est justement ce qui en fait une discipline vivante, ce qui est stimulant car bien plus que l'éclatement, le danger pour la discipline, c'est la routine, tout ce qui contribue à la figer, à la définir une fois pour toutes. 


\section{NOTES}

1. Français, Lectures et Expression - $3^{\mathrm{e}}$. Paris, Belin, 1999.

2. DEVELAY M. :L'apprentissage à l'enseignement.1992.

3. Rappelons que le concours de recrutement des professeurs des écoles dans sa définition actuelle ne comporte pas de littérature. Le projet pour 2003 confirme cet état de fait en dépit de la place faite à la littérature dans les nouveaux programmes de l'école primaire.

Notons également que, dans l'esprit des élèves, la notion de discipline se dessine plus nettement au collège ou elle est prise en charge par un enseignant spécialiste.

4. NÉE P. : Lire Nadja de Breton.Paris, Dunod, 1993.

5. MAISONNEUVE L. :Apprentissage de la lecture. Méthodes et manuels.Rennes, Université de Rennes II, décembre 2001.

6. DENIZEAU M.-T. : « Les textes littéraires dans les manuels de lecture à l'école élémentaire », in Enseigner la littérature, (Éds : Fourtanier M.-J., Langlade G.). Delagrave - CRDP Midi-Pyrénées, p. 193: "Il(le traitement du texte) se limite à une lecture tournante, à un résumé oral du sens, à la demande d'explication de mots difficiles avec dictionnaire et surtout de plus en plus à une fiche de lecture individuelle où les questions sur le sens (vrai/faux), sur le vocabulaire, ne demandent pas la présence du maître."

7. TAUVERON C. : «Approche de la littérature à l'école élémentaire », inRecherches en didactique de la littérature, rencontres de Rennes,mars 2000, (Éds : Fourtanier M.J., Langlade G., Rouxel A.). Rennes, PUR, 2001, pp. 127-128: «Au-delà du cours préparatoire, on croit aider à la compréhension, en éliminant d'abord ce qu'on pense qui lui fait obstacle (on explique prealablement les mots difficiles), en exigeant ensuite la restitution du contenu littéral(par l'intermédiaire de questions appelant des relevés précis dans le texte).»

8. Voir à ce propos les analyses de Jean-François Massol : « La lecture des textes littéraires, des images, du spectacle vivant: éléments de problématique et questions ", in Recherches en didactique de la littérature. Op. cit.,pp. 105-109.

9. FOURTANIER M.-J.: Les Mythes dans l'enseignement du français. Paris, Bertrand-Lacoste, 1999.

\section{RÉSUMÉS}

Non disponible

Not Available 
AUTEUR

ANNIE ROUXEL

Université Rennes II, IUFM de Bretagne 